\title{
IS AN EXTERNAL FOCUS OF ATTENTION MORE BENEFICIAL THAN AN INTERNAL FOCUS TO BALL CATCHING IN CHILDREN?
}

\author{
Reza Abdollahipour and Rudolf Psotta \\ Department of Natural Sciences in Kinanthropology, Faculty of Physical Culture, \\ Palacky University Olomouc, Olomouc, Czech Republic
}

Original scientific paper

UDC: $159.952-053.2: 796.012 .576$

\begin{abstract}
:
The performance benefits of adopting an external relative to an internal focus of attention have been demonstrated for many different targeting and balance tasks. No study has examined attentional focus effects for interceptive motor skills. Also, the majority of studies have used adult participants. In this study, children (mean age: 8.75 years, $\mathrm{SD}=0.79 ; 15$ girls, 9 boys) were required to catch tennis balls in the frontal plane under the external focus (EF), internal focus (IF), and control (Cont) conditions. Participants were asked to stand behind a yellow line $(2 \times 100 \mathrm{~cm}), 15 \mathrm{~m}$ apart from a tennis ball throwing machine. In a within-participant design, participants performed 10 trials under each IF ("concentrate on your hands"), EF ("concentrate on the ball"), or Cont (no focus instructions) conditions. The order of conditions was counterbalanced. Performance data were analyzed using a one-way analysis of variance (ANOVA) with repeated measures on attentional focus conditions (internal, external, and control) (alpha $=.05)$. Catching performance was significantly different and more effective in the EF $(M=1.53, S D=0.25)$ than in the IF $(M=1.39, S D=0.35)$ condition, while both EF and IF were not significantly different from the Cont $(M=1.49, S D=0.28)$ condition. The current findings suggest that the external focus of attention is more beneficial, compared to the internal focus, to motor performance of an interceptive skill in children.
\end{abstract}

Key words: attentional focus, instructions, motor performance, interceptive skills

\section{Introduction}

In order to enhance performance and learning of motor skills, verbal instructions and augmented feedback are probably the most important forms of information that direct performer's focus of attention to specific aspects of a motor task. Current studies on attentional focus instructions suggest that the optimal performance outcome can be reached when the performer's concentration is directed to the movement effects, i.e., an external focus (EF) rather than body movements, i.e., internal focus (IF) (see Wulf, 2007, 2013; Wulf \& Lewthwaite, 2016). To explain this notion, it has been proposed that an external focus of attention on the intended movement effects promotes concentration on the taskgoal, which impacts the optimization of goal-action coupling (Wulf \& Lewthwaite, 2016). More specifically, as it has been suggested by the constrained action hypothesis (Wulf, McNevin, \& Shea, 2001) about the underlying mechanisms of attentional focus effects on motor control, concentration on the intended movement effects or object (EF) decreases the performer's conscious processes with a consequence of facilitation in automatic control processes associated with neuromuscular coordination. In contrast, concentration on bodily movements (IF) raises the performer's conscious processes with the increased chance for interruption in automatic motor control processes.

Studies have supported the constrained action hypothesis by showing that indicators of more advanced motor performance were found under $\mathrm{EF}$ rather than IF. For example, it has been shown that EF has increased movement fluency and regulation as indicators of movement automaticity (Kal, van der Kamp, \& Houdijk, 2013), correlations among body dimensions with the consequence of reduced variability in the performance outcome (Lohse, Jones, Healy, \& Sherwood, 2014), frequency of movement regulation that indicates higher contribution of reflexes (e.g., McNevin, Shea, \& Wulf, 2003), optimal and economical use of muscular activities (e.g., Lohse \& Sherwood, 2012; Marchant, Greig, \& Scott, 2009), and instant modulation of 
intracortical inhibition within the primary motor cortex (Kuhn, Keller, Ruffieux, \& Taube, 2016). The evidence shows that EF accelerates the rate of movement coordination with a consequence of optimizing the task outcome, whereas IF decelerates progress of movement coordination with a consequence of preventing performers from achieving the optimal task outcome.

Numerous pieces of research on attentional focus instructions have supported the superiority of EF over IF of attention for performance and learning of a variety of targeting and balance motor tasks in novice adults (for a review see Wulf, 2013), and in typical children (Hadler, Chiviacowsky, Wulf, \& Schild, 2014; Perreault \& French, 2015; Wulf, Chiviacowsky, Schiller, \& Ávila, 2010). In the current research we chose an interceptive motor task, i.e., two-handed catching task since there is no study about the effects of attentional focus instructions on interceptive motor skills (i.e., catching) in children. Particularly, for a successful catch the performer needs to predict the optimal time to move and adjust his/her hands' position in time to conform to the trajectory of the ball (Gentile, 2000). Also, it should be noted that in catching both visual and movement control processes should be continuously updated according to spatial-temporal conditions of the object (Vickers, 2007). Moreover, a functional bimanual co-ordination of the movements of the hands is necessary due to a high demand for coordination of various degrees of freedom (Davids, 2002; Tayler \& Davids, 1997). These highlight a relatively higher demand on adaptation of spatial-temporal movement coordination that occurs with rapid adjustments in motor commands in the process of action planning and programming (Gentile, 2000; Magill, Chamberlin, \& Hall, 1991). These adjustments in the process of action planning and programming may rapidly impact relocation of the performer's attention (Hassan, Dowling, \& McConkey, 2014; van der Kamp \& Renshaw, 2015) and consequently the performance outcome.

It should be pointed out that the targeting skills such as basketball free throw (Perreault \& French, 2015), soccer throw-in task (Wulf, et al., 2010), forehand tennis strokes (Hadler, et al., 2014), and balance motor skills such as riding a pedalo (Flores, Gomes Schild, \& Chiviacowsky, 2015) are predominantly controlled by either predictive or prospective control systems. However, during performance of interceptive motor skills (i.e., catching), a simultaneous combination of both predictive and prospective control systems is necessary for optimal performance of the action (Panchuk \& Vickers, 2009). Therefore, it is questionable which type of attentional focus instructions is more beneficial to the motor tasks that require a rapid adaptation of motor commands for selection of the appropriate motor response (i.e., catching).
Catching is a complex fundamental developmental motor skill that influences involvement of children in sport games during childhood (Heywood \& Getchell, 2014). Therefore, providing appropriate instructions for children facilitates performance and learning of catching which leads them to a more active life. Thus, the purpose of the present study was to examine the effects of different attentional focus instructions on execution of an interceptive motor task in school-age children. Specifically, we were interested in understanding whether the beneficial effects of external compared to internal attentional focus instructions could also be found in the performance of a catching task in typically developing children. In the current research we assumed that for an optimized perception-action coupling and successful catching performance, the instructions that direct the attentional focus of performers to the object (e.g. ball) would be more beneficial than the instructions that direct attentional focus of performers to coordination of bodily movement patterns (e.g. hands) in children.

\section{Methods}

\section{Participants}

Twenty-four children, including 15 girls and nine boys (mean age: $8.8 \pm 0.8$ years) participated in the study. The participants were selected from mainstream elementary schools of Czech Republic that adopted the same National Education Curriculum including the program for physical education (PE). Before the catching task, the PE teachers carried out the screening of potential participants. Only the children whose performance grades during the PE classes were below the average grade of the education system were selected for the study. Although children may have had previous experience with similar tasks, they did not have any experience with the task used in this study. Also, the check list of movement assessment battery for children - second version (Henderson, Sugden, \& Barnett, 2007) for the age band 7-10 was used by PE teachers to assess the level of motor skills, and screening of potential motor impairments. According to the traffic light system in each age band, only children who were classified in the green zone indicating no detected movement difficulty were included in this study. Moreover, only healthy children without general medical diseases or neurological dysfunction, perceptual, mental and physical disabilities, developmental coordination disorders and attention deficit hyperactivity disorder were included in the study. Children were not aware of the purpose of the study. The study was approved by the institutional ethical review board of the university. Informed consent was obtained from the school principals and children's parents. 


\section{Apparatus and task}

The task was two-handed catching of the tennis balls thrown by a tennis ball machine (Lobster Elite Grand 4, Lobster Sports, Inc., North Hollywood, CA, USA). Children were asked to catch tennis balls in the frontal plane. Each child completed 30 catching trials in three blocks of ten trials under each attentional focus condition in the gym hall. There was a 15 -second break between trials during which participants received one of the attentional focus instructions. To avoid the effects of mental and/or physical fatigue on the performance a 1-minute break was applied between each block of 10 trials. The software of the tennis ball machine was adjusted to throw tennis balls at the same angle of $40^{\circ}$ at the moment of release at an initial height of $30 \mathrm{~cm}$ from the ground providing the balls arrived at the level of the participant's chest area (see Figure 1).

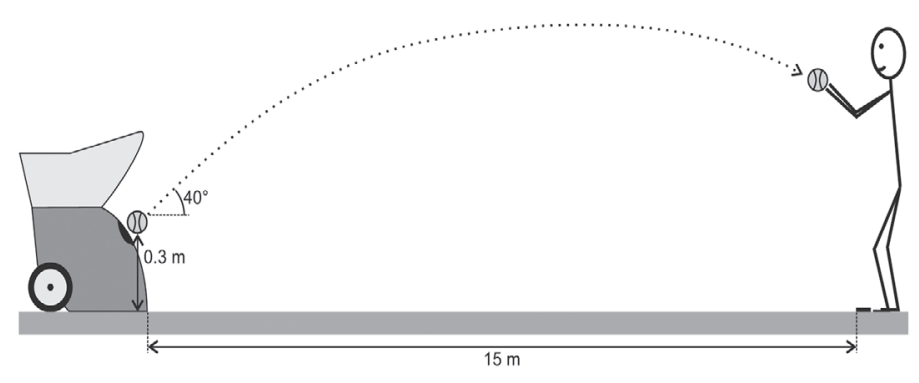

Figure 1. The schematic presentation of catching task.

Ejection speed of the balls was set at 26.00 $\mathrm{mph}$ at the moment of release from the machine. To secure accuracy of the tennis ball machine, velocity of the balls thrown by the tennis machine was measured by a radar gun (Stalker Radar, Applied Concepts, Inc., Dallas, TX, USA) before the experiments. The results showed that the average speed of the balls at the moment of release was $26.00 \pm 0.94$ $\mathrm{mph}$. The pilot results showed the accuracy of ball machine within a circular area with radius of $0.3 \mathrm{~m}$ in the frontal plane. A specific sound was made by the machine when each ball was thrown from the machine. This condition was identical for all trials and attentional focus conditions. All the catching trials were recorded using two cameras (Panasonic HDC-TM900, Panasonic, Kadoma, Japan) at a frequency of $50 \mathrm{~Hz}$ positioned $3 \mathrm{~m}$ to the left and right sides of participants. The cameras were mounted on tripods at a height proportional to the shoulder height of the participants.

\section{Procedure}

At the beginning of the experiment children were asked to stand behind a yellow line $(2 \mathrm{~cm}$ in width $\times 100 \mathrm{~cm}$ in length) that was located $15 \mathrm{~m}$ away from the tennis ball machine and to be ready to catch the ball. The correct form of the catching task was demonstrated to the participants by the experimenter. Each participant performed two practice trials to become familiar with the task. Then, in a within-subject design, they were required to perform 10 trials under the IF ("Concentrate on your hands"), EF ("Concentrate on the ball"), or Cont (no focus instructions) conditions. It is necessary to highlight that children were not asked for having visual fixation on the ball under the EF instructions. Also, in case participants were looking at their hands under the IF condition, they were told not to look at their hands to ensure that any effect of IF is due to attention and not to interference with visual processes required for catching the ball. The order of the focus conditions for execution of catching task was counterbalanced.

Interviews were carried out with each child after each block of ten trials for each focus condition and the answers were recorded. All the children were asked two questions after 10 trials completed under either the EF or IF condition. The first question for the EF condition was "Did you concentrate on the ball?" and for the IF condition was "Did you concentrate on your hands"? The first question for the Cont condition was "What did you concentrate on?" The second question for all attentional focus conditions was "On a scale from 1 (not at all) to 5 (extremely), to what extent did you concentrate?" The answers were analyzed to identify to what extent the participants adopted the attentional focus instructions.

\section{Dependent variables}

The dependent variable was children's motor performance of catching indicated by the mean score out of ten catches. The child's performance was assessed by two independent researchers via video analysis according to three criteria used by Cesqui, d'Avella, Portone, and Lacquaniti (2012). Specifically, 2 points were awarded for a definite catch of the ball with both hands without the ball falling from the hands; 1 point was given when the ball was touched with the hand(s) but not caught; and 0 point was given for no contact between the hand and the ball. The ratings were compared by the first author subsequently. In the case of two-interrater discrepancy of scores in each trial, the raters were asked to re-analyze a given video sequence and achieve a consensus. The kappa coefficient showed a high inter-rater agreement between the two researchers $(\kappa=.926)$. The researchers in the role of raters were not aware of the purpose of the study nor of the attentional focus conditions. 


\section{Data analysis}

To measure movement functionality associated with catching performance, the mean scores of catching performance across trials were calculated as the performance outcome for each participant in the different attentional focus conditions. In the first step, the Shapiro-Wilks test was used for the assessment of data distribution normality. The results showed that mean scores of catches were distributed normally for all attentional focus conditions.

The data were analyzed using a one-way analysis of variance (ANOVA) with repeated measures on attentional focus conditions (IF, EF, and Cont). The results of Mauchly's test for the evaluation of the sphericity assumption showed that there was no violation for the assumption. To test all posthoc comparisons, the Bonferroni adjustments were used. Estimates of effect size were calculated using two measures. First, partial eta squared $\left(\eta_{\mathrm{p}}{ }^{2}\right)$ was utilized (Larson-Hall, 2009). Then, Cohen's $d$ was employed as a measure of the difference between focus conditions in within-subject designs that also took into account the correlation between the two means (Morris \& DeShon, 2002). For all the statistical tests the significance was set at the level of $\alpha=.05$. Data were analyzed using SPSS 21 .

\section{Results}

The results of the post-performance interviews revealed that all the children (100.0\%) in the EF and IF conditions reported adaptation to attentional focus instructions. More specifically, children stated a relatively high rate of adherence to the external ( $4.42 \pm 0.97$ points, corresponding to $88.4 \%$ ) and internal ( $4.08 \pm 0.88$ points, corresponding to $81.6 \%$ ) focus instructions. In the Cont condition, most participants $(75.0 \%)$ reported that they focused on the ball ( $4.00 \pm 0.70$ points), and some of them $(16.6 \%)$ stated the preference of focusing on their hands ( $4.00 \pm 0.00$ points), or a few of them (8.4\%) declared focusing on both the ball and hands

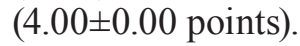

Figure 2 shows the catching performance for the children across trials under the different attentional focus conditions. The results revealed that the main effect of attentional focus condition, $\mathrm{F}(2$, $46)=3.508, \mathrm{p}=.038, \eta_{\mathrm{p}}{ }^{2}=.132$ was significant. Posthoc analysis showed that the children's catching performance was significantly more successful in the $\mathrm{EF}(\mathrm{M}=1.53, \mathrm{SD}=0.25)$ than in the IF $(\mathrm{M}=1.39$, $\mathrm{SD}=0.35)$ condition $(p=.026, d=0.62)$. There was no significant difference between the Cont condition $(\mathrm{M}=1.49, \mathrm{SD}=0.28)$ and either $\mathrm{EF}(\mathrm{p}>.99, \mathrm{~d}=0.16)$ or IF conditions $(\mathrm{p}=.395, \mathrm{~d}=0.31)$.

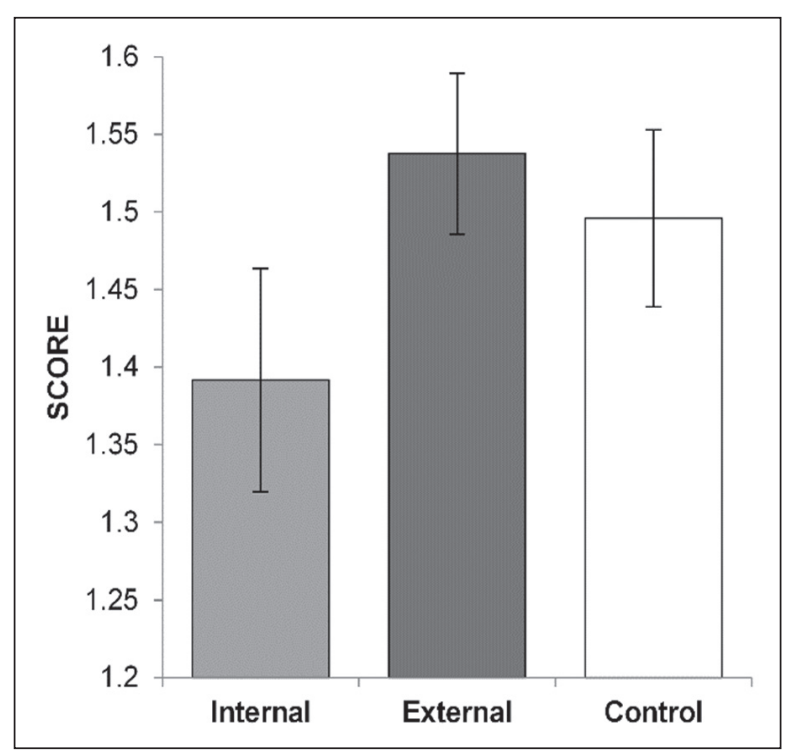

Figure 2. Means of catching scores for the internal focus, external focus and control conditions. Note: Error bars represent standard errors.

\section{Discussion and conclusions}

The beneficial effects of external versus internal attentional focus instructions have been shown in adults and children in a variety of targeting and balance motor skills (for a review see Wulf, 2013; Wulf \& Lewthwaite, 2016). The present study showed that these performance advantages could also be found for interceptive timing motor skills (e.g., catching task) in children. Therefore, the higher beneficial effect of EF rather than IF is not only for targeting and balance motor tasks (Flores, et al., 2015; Hadler, et al., 2014; Perreault \& French, 2015, Wulf, et al., 2010), which are predominately controlled by either predictive or prospective control systems, but also for interceptive motor skills (i.e., catching), in which a simultaneous combination of both systems is necessary for optimal action of planning and programming (Panchuk \& Vickers, 2009). That is, interceptive motor tasks (e.g., catching) that have a higher demand for updating motor commands and co-ordination of various degrees of freedom (Davids, 2002; Sarlegna \& Mutha, 2015; Tayler \& Davids, 1997) could also benefit from EF compared to IF. It seems that EF compared to IF helps the motor system to co-ordinate the degrees of freedom more effectively to choose and produce an appropriate and optimal response. One possible explanation for these results might be due to impact of concentration on the intended movement effects in the $\mathrm{EF}$ that increases automatic control processes during action planning to facilitate functional linkage of goalaction coupling (Wulf, 2013; Wulf, et al., 2001). 
This study also highlights that performance of young school-age children, who are typically at the early stages of developing their expertise level, could be enhanced more effectively under the EF than under the IF instructions. Therefore, not only have novice adults benefited from EF instructions (for a review see Wulf, 2013), but children also (Hadler, et al., 2014; Perreault \& French, 2015; Wulf, et al., 2010; present study). These results suggest that young children who are in the process of developing their fundamental motor skills could benefit from advantages of the EF instructions compared to the IF instructions (Emanuel, Jarus, \& Bart, 2008; Peh, Chow, \& Davids, 2011).

Another interesting result of this study, in comparison to previous studies on children (Emanuel, et al., 2008; Hadler, et al., 2014; Wulf, et al., 2010), was obtained from the retrospective interviews after the Cont condition. The results showed that when left to their own decisions (Cont), $75 \%$ of children adopted an attentional focus on the ball to perform this interceptive motor task. These results are not surprising as it could be due to the (target-oriented) nature of the task. It should be noted that even though most of the participants in the Cont condition reported adopting EF, performance outcome in the Cont condition was not as effective as it was in the EF condition compared to the IF condition. Therefore, promoting the EF instructions was more beneficial than the IF instructions to reaching the optimal performance in children. These results suggest that attentional focusing on the flying ball may be crucial for successful catching.

An alternative suggestion for the explanation of advantages of EF over IF in the present study might be due to the enrichment of perceiving visual information in EF compared to IF. In other words, adopting the internal attentional focus instructions may worsen attunement to visual information processing of a performer's visual perception system in interceptive motor skills. However, this study does not bring a direct evidence of the hypothesis on the functional association between the attentional focus on an action and visual attention. Despite that, it should be noted that previous studies have shown that the underlying mechanisms causing the beneficial effects of the EF instructions are independent of vision for discrete motor actions such as aiming or jumping (Abdollahipour, Psotta, \& Land, 2016; Land, Tenenbaum, Ward, \& Marquardt, 2013; Schlesinger, Porter, \& Russell, 2013; Sherwood, Lohse, \& Healy, 2014), which typically use feedforward control system. It is important to highlight that interceptive motor skills (i.e., catching), are highly dependent on the visual information processing for the co-ordination of movement skills (Davids, 2002) and on the anticipation of the contact time with an object, "typically use feed-forward and feedback control system (Gentile, 2000; Magill, et al., 1991). Therefore, future studies should investigate the role of vision and attentional focus instructions in performance of motor tasks motor control of which depends on feedback control system.

The current results appear to be the first to provide additional support for the constrained action hypothesis (Wulf, et al., 2001) as regards interceptive motor skills, underpinning the idea that IF of attention increases focusing on the self with the consequence of increasing conscious control processes and debilitating performance (McKay, Lewthwaite, Wulf, \& Nordin, 2015), whereas an EF of attention enhances performance by decreasing the focus on the self (Wulf \& Lewthwaite, 2010).

In short, as with other targeting and balance motor tasks (Flores, et al., 2015; Hadler, et al., 2014; Perreault \& French, 2015; Wulf, et al., 2010), performance of young school-age children in an interceptive motor skill could also benefit from EF compared to IF of attention. Specifically, the findings suggest that the external attentional focus on the ball appears to be more beneficial to successful performance of the catching task than the internal focus on hands' movements. This study suggests that concentration on the movement effects could be more effective for optimal performance of interceptive motor skills (i.e., catching) than the conscious concentration on body movements. The results presented in this study suggest that coaches and PE teachers should use the EF instruction, i.e., attentional focus on the movement effects, rather than the IF instructions related to movement techniques for teaching interceptive motor tasks.

\section{References}

Abdollahipour, R., Psotta, R., \& Land, W. (2016). The influence of attentional focus instructions and vision on jump height performance. Research Quarterly for Exercise and Sport, 87, 408-413.

Cesqui, B., d'Avella, A., Portone, A., \& Lacquaniti, F. (2012). Catching a ball at the right time and place: Individual factors matter. PLoS ONE, 7, e31770.

Davids, K. (2002). Interceptive actions in sport: Information and movement. London: Routledge.

Emanuel, M., Jarus, T., \& Bart, O. (2008). Effect of focus of attention and age on motor acquisition, retention, and transfer: A randomized trial. Physical Therapy, 88, 251-260. 
Fabrice, R., Sarlegna, F.R., \& Mutha, P.K. (2015). The influence of visual target information on the online control of movements. Vision Research, 110, 144-154.

Flores, F.S., Gomes Schild, J.F., \& Chiviacowsky, S. (2015). Benefits of external focus instructions on the learning of a balance task in children of different ages. International Journal of Sport Psychology, 46, 311-320.

Gentile, A.M. (2000). Skill acquisition: Action, movement, and neuromotor processes. In J.H. Carr \& R.D. Shepherd (Eds.), Movement science: Foundations for physical therapy ( $2^{\text {nd }}$ ed.; pp. 111-187). Rockville, MD: Aspen.

Hadler, R., Chiviacowsky, S., Wulf, G., \& Schild, J.F.G. (2014). Children's learning of tennis skills is facilitated by external focus instructions. Motriz: Revista De Educacao Fisica, 20, 418-422.

Hassan, D., Dowling, S., \& McConkey, R. (2014). Sport, coaching and intellectual disability. London: Routledge.

Henderson, S.E., Sugden, D.A., \& Barnett, A. (2007). Movement Assessment Battery for Children-2. $2^{\text {nd }}$ edition (Movement ABC-2). Examiner's manual. London: Pearson Assessment.

Heywood, K., \& Getchell, N. (2014). Life span motor development ( $6^{\text {th }}$ ed.). Champaign, IL: Human Kinetics.

Kal, E., van der Kamp, J., \& Houdijk, H. (2013). External attentional focus enhances movement automatization: A comprehensive test of the constrained action hypothesis. Human Movement Science, 32, 527-539.

Kuhn, Y.A., Keller, M., Ruffieux, J., \& Taube, W. (2016). Adopting an external focus of attention alters intracortical inhibition within the primary motor cortex. Acta Physiologica. Advance online publication. doi:10.1111/ apha.12807

Land, W.M., Tenenbaum, G., Ward, P., \& Marquardt, C. (2013). Examination of visual information as a mediator of external focus benefits. Journal of Sport and Exercise Psychology, 35, 250-259.

Larson-Hall, J. (2009). A guide to doing statistics in second language research using SPSS. New York, NY: Routledge.

Lohse, K.R., Jones, M.C., Healy, A.F., \& Sherwood, D.E. (2014). The role of attention in motor control. Journal of Experimental Psychology: General, 143, 930-948.

Lohse, K.R., \& Sherwood, D.E. (2012). Thinking about muscles: The neuromuscular effects of attentional focus on accuracy and fatigue. Acta Psychologica, 140, 236-245.

Magill, R.A., Chamberlin, C.J., \& Hall, K.G. (1991). Verbal knowledge of results as redundant information for learning an anticipation timing skill. Human Movement Science, 10, 485-507.

Marchant, D.C., Greig, M., \& Scott, C. (2009). Attentional focusing instructions influence force production and muscular activity during isokinetic elbow flexions. Journal of Strength and Conditioning Research, 23, 2358-2366.

McKay, B., Lewthwaite, R., Wulf, G., \& Nordin, A. (2015). The self: Your own worst enemy? A test of the self-invoking trigger hypothesis. Quarterly Journal of Experimental Psychology, 68, 1910-1919.

McNevin, N.H., Shea, C.H., \& Wulf, G. (2003). Increasing the distance of an external focus of attention enhances learning. Psychological Research, 67, 22-29.

Morris, S.B., \& DeShon, R.P. (2002). Combining effect size estimates in meta-analysis with repeated measures and independent-groups designs. Psychological Methods, 7, 105-125.

Panchuk, D., \& Vickers, J.N. (2009). Using spatial occlusion to explore the control strategies used in rapid interceptive actions: Predictive or prospective control? Journal of Sports Sciences, 27, 1249-1260.

Peh, S.Y.C., Chow, J.Y., \& Davids, K. (2011). Focus of attention and its impact on movement behaviour. Journal of Science and Medicine in Sport, 14, 70-78.

Perreault, M.E., \& French, K.E. (2015). External-focus feedback benefits free-throw learning in children. Research Quarterly for Exercise and Sport, 86, 422-427.

Sarlegna, F., \& Mutha, P.K. (2015). The influence of visual target information on the online control of movements. Vision Research, 110, 144-154.

Schlesinger, M., Porter, J., \& Russell, R. (2013). An external focus of attention enhances manual tracking of occluded and visible targets. Frontiers in Psychology, 3, 591.

Sherwood, D.E., Lohse, K.R., \& Healy, A.F. (2014). Judging joint angles and movement outcome: Shifting the focus of attention in dart-throwing. Journal of Experimental Psychology: Human Perception and Performance, 40, 1903-1914.

Tayler, M.A., \& Davids, K. (1997). Catching with both hands: An evaluation of neural cross-talk and coordinative structure models of bimanual coordination. Journal of Motor Behavior, 29, 254-262.

van der Kamp, J., \& Renshaw, I. (2015). Information-movement coupling as a hallmark of sport exercise. In J. Baker \& D. Farrow (Eds.), Routledge handbook of sport expertise (pp. 50-63). London: Routledge.

Vickers, J.N. (2007). Perception, cognition and decision training: The quiet eye in action. Champaign, IL: Human Kinetics.

Wulf, G. (2007). Attention and motor skill learning. Champaign, IL: Human Kinetics.

Wulf, G. (2013). Attentional focus and motor learning: A review of 15 years. International Review of Sport and Exercise Psychology, 6, 77-104.

Wulf, G., Chiviacowsky, S., Schiller, E., \& Ávila, L.T. (2010). Frequent external-focus feedback enhances motor learning. Frontiers in Psychology, 1, 190. 
Wulf, G., \& Lewthwaite, R. (2010). Effortless motor learning? An external focus of attention enhances movement effectiveness and efficiency. In B. Bruya (Ed.), Effortless attention: A new perspective in attention and action ( $\mathrm{pp}$. 75-101). Cambridge, MA: MIT Press.

Wulf, G., \& Lewthwaite, R. (2016). Optimizing performance through intrinsic motivation and attention for learning: The OPTIMAL theory of motor learning. Psychonomic Bulletin \& Review, 23, 1382-1414.

Wulf, G., McNevin, N., \& Shea, C.H. (2001). The automaticity of complex motor skill learning as a function of attentional focus. Quarterly Journal of Experimental Psychology Section A: Human Experimental Psychology, $54,1143-1154$.

Submitted: November 5, 2016

Accepted: January 30, 2017

Published Online First: September 29, 2017

Correspondence to:

Reza Abdollahipour

Department of Natural Sciences in Kinanthropology

Faculty of Physical Culture, Palacky University

Olomouc

třída Míru 117, 77111 Olomouc, Czech Republic

Phone: +420585636100

Fax: +420 585412899

E-mail: reza.abdollahipour@upol.cz

\section{Acknowledgment}

The study was supported by the Czech Science Foundation (GAČR) under the project No. GA 16-17945S, and the Internal Grant Agency of the Palacky University Olomouc under the project No. IGA_FTK_2017_008. 